\title{
Comparison of the greenhouse effect between Earth and Venus using multiple atmospheric layer models
}

\author{
William Song Liu ${ }^{1, *}$ \\ ${ }^{1}$ Stanford Online High School, Redwood City, CA, United States
}

\begin{abstract}
To better understand the mechanisms of global warming, we developed a one atmospheric layer model for Earth and a multiple atmospheric layer model $(\mathrm{N}=111)$ for Venus. Earth's greenhouse gas atmosphere has an average of $78.9 \%$ absorption efficiency of terrestrial radiation $(f=0.789)$, while we assume Venus' atmosphere has a near $100 \%$ absorption efficiency ( $f=1)$ due to its denser, CO2-rich atmosphere. Viewing the atmospheric layers as blackbodies, we modeled the surface temperature of Earth and Venus, both of which are able to predict the respective actual planetary temperatures. The consistency $(\delta<1 \%)$ between the modeled surface temperature and the observed surface temperature of these two planets suggest that the multiple layer greenhouse gas atmosphere mechanism could explain Venus' runaway global warming and scorching temperature. The results of these two models suggest that if Earth continues to experience uncontrolled greenhouse gas emissions, global warming and its negative outcomes may be further exacerbated.
\end{abstract}

\section{Introduction}

The greenhouse effect describes the phenomenon of a planet's atmosphere being able to retain heat that would have been reflected or emitted into space, resulting in the planet surface being at a higher temperature than it would have without an atmosphere. Earth, as of 2017, has an average surface temperature of $15{ }^{\circ} \mathrm{C}[1]$, while it would have a predicted temperature of $-18{ }^{\circ} \mathrm{C}$ without an atmosphere.

The sun emits electromagnetic radiation, mainly in the ultraviolet and visible wavelengths, in all directions. The inverse square law can model the amount of solar radiation Earth is receiving based on the distance between the Sun and the Earth and the radius of the Earth, which computes to being $343 \mathrm{~W} / \mathrm{m}^{2} .103 \mathrm{~W} / \mathrm{m}^{2}$, or approximately $30 \%$ of the solar radiation that Earth receives, is directly reflected back into space by the atmosphere. Most of the radiation that is not reflected by the atmosphere is either absorbed by the atmosphere or absorbed by the surface of the Earth. Since Earth's global temperature is not increasing as rapidly as the amount of electromagnetic radiation it is receiving, Earth must be emitting a nearly equivalent amount of electromagnetic radiation into space, which is primarily in the form of infrared radiation, or heat. Some of the heat that is reemitted by the surface of the Earth comes into contact with greenhouse gasses in the atmosphere, which are gasses that are also able to absorb and reemit heat, effectively preventing some portion of the heat in the atmosphere from escaping.

The most potent greenhouse gasses that exist in the troposphere are water vapor, carbon dioxide, methane, nitrous oxide, chlorofluorocarbons, and ozone. Water vapor is generally ignored in discussions of anthropogenic causes of global warming, as variations in the amount of atmospheric water vapor are not known to be directly influenced by human activity. The other listed greenhouse gasses are known to be either anthropogenically emitted, or in the case of ozone, its formation is catalyzed by other anthropogenically emitted gasses. While the atmospheric content of these greenhouse gasses is minor, as they make up less than $1 \%$ of the atmosphere, an increase in the concentration of greenhouse gasses result in significant differences.

As the concentrations of these greenhouse gasses change, the amount of heat the atmosphere is able to absorb also changes. Over the last century, there have been minor decreases and major increases in the global temperature. The net increase in the global temperature is often referred to as global warming. There has been debate over whether global temperature variations are really caused by anthropogenic activity, as natural causes of global temperature changes include volcanic activity in the short term, and changes in the orbit of the Earth, changes in the tilt of the Earth, and variation in solar activity in the form of sunspots in the long term. However, the uncertainty has mostly been cleared up with better measurements and models of atmospheric concentrations of greenhouse gasses in relation to the global temperature [2].

Earth and Venus are very similar in primary properties [3], in terms of both having "atmospheres, weathered surfaces, massive volcanoes, and chemically and thermally evolved interiors" [4]. We know from empirical data that Venus has a significantly higher surface temperature than Earth, which is caused by a larger greenhouse effect. To

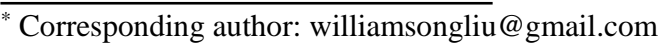


better understand the greenhouse effect mechanism and the projections of global warming, atmospheric layer models were developed for Earth and Venus.

We will begin by examining the simplest atmospheric model, the one layer model. Through modeling the atmosphere as a single object that is able to absorb and emit long-wave radiation, with the emissions being both towards and away from the planet, it is able to accurately predict the surface temperature of Earth but not Venus. This paper then proposes a multiple atmospheric layer model that is suitable for modeling Venus' atmosphere, and demonstrates that the number of layers can be adjusted to accurately predict the surface temperature of Venus.

\section{Methods}

\subsection{One atmospheric layer model}

The planetary effective temperature model, also known as the planetary energy balance model, is based on the principle that the energy absorbed by a planet equals the energy emitted by a planet. This balance must be true, otherwise, the planet would be constantly gaining energy or losing energy, which is not the case. To incorporate the effects of the atmosphere, a one-layer approximation can be used, treating the atmosphere as having a single layer that absorbs and emits radiation (Figure 1).

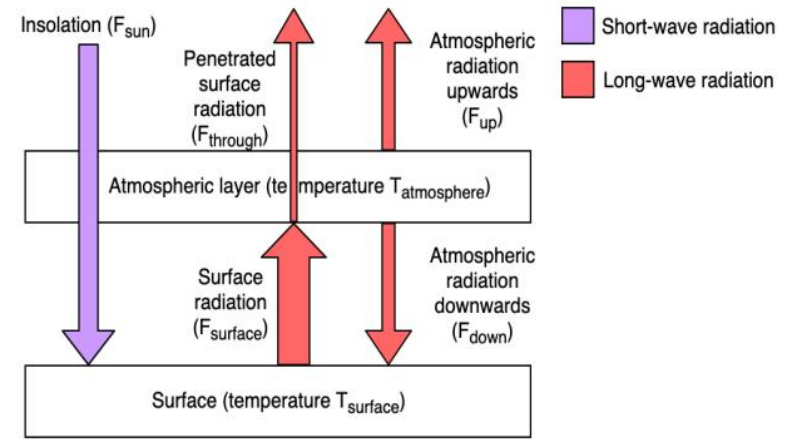

Fig. 1. A one atmospheric layer model of a planet at planetary energy balance. The greenhouse atmosphere is transparent to insolation, while it absorbs long-wave planetary radiation at an efficiency of $\mathrm{f}$.

Likewise, energy balance is also a fundamental principle in the one atmospheric layer model. The surface, the atmosphere, and the Earth as a whole can be isolated, and all of them maintain a constant temperature.

This model is developed with a few simple assumptions, including (1) all of the short-wave solar radiation penetrates the atmospheric layer; (2) a fraction $f$ of the long-wave surface radiation is absorbed by the atmospheric layer, while a fraction $(1-f)$ penetrates the atmospheric layer and goes into space; and (3) $F_{u p}=F_{\text {down }}$ as the atmospheric layer should emit an equal amount of radiation in both directions.

Based on the energy balance principle, the following equations can be created:

$$
F_{\text {sun }}=F_{\text {through }}+F_{\text {up }}
$$

$$
\begin{gathered}
F_{\text {surface }}-F_{\text {through }}=F_{\text {up }}+F_{\text {down }} \\
F_{\text {sun }}+F_{\text {down }}=F_{\text {surface }}
\end{gathered}
$$

Each of these terms is defined:

- $F_{\text {sun }}=\frac{S * \pi r^{2} *(1-\alpha)}{4 \pi r^{2}}=\frac{S(1-\alpha)}{4}$, where $\mathrm{S}$ is the solar flux of the planet, $\mathrm{r}$ is the radius, and $\alpha$ is the average albedo.

- $F_{\text {surface }}=\sigma T_{\text {surface }}^{4}$, a direct application of the StefanBoltzmann law.

- $F_{\text {through }}=(1-f) * F_{\text {surface }}=(1-f) * \sigma T_{\text {surface }}^{4}$, the fraction of radiation that penetrates the atmosphere.

- $F_{\text {up }}=F_{\text {down }}=f \sigma T_{\text {atmosphere }}^{4}$, the fraction of radiation absorbed by the atmosphere and then emitted.

Finally, solving for $\mathrm{T}_{\text {surface}}$, the model is obtained [5]:

$$
T_{\text {surface }}=\sqrt[4]{\frac{s(1-\alpha)}{4 \sigma\left(1-\frac{f}{2}\right)}}
$$

\subsection{Multiple atmospheric layer model (Venus)}

Venus has a significantly more massive atmosphere, creating an atmospheric pressure almost 91 times as large as Earth's. In addition, Venus' atmospheric composition is $96.5 \%$ carbon dioxide, as opposed to all greenhouse gasses being trace gasses in Earth's atmosphere, where Earth's carbon dioxide concentration is only $410 \mathrm{ppm}(0.041 \%)$. While the one atmospheric layer model did serve as a reasonable approximation for Earth's atmosphere, is not able to accurately model Venus' atmosphere due to two factors described above. Instead, it is more suitable to divide Venus "thick" atmosphere into layers and model them using a multiple atmospheric layers model.

This model is developed with some of the same assumptions, including (1) the short-wave solar radiation penetrates all of the atmospheric layers without any portion of it being absorbed; (2) unlike Earth's atmosphere where a fraction $(1-f)$ of the long-wave radiation is able to directly penetrate the atmospheric layer, nearly all of the long-wave radiation will be absorbed by every atmospheric layer, due to Venus' extremely high concentration of carbon dioxide. Thus, we will assume $\mathrm{f}=$ $100 \%$; (3) each atmospheric layer emits an equal amount of long-wave radiation in the up and down directions.

As shown in Figure 2, $\mathrm{N}$ represents the number of layers the atmospheric model has, and $\mathrm{M}$ represents a layer within the atmosphere that is not the Nth layer or the surface $(0<\mathrm{M}<\mathrm{N})$. Similar to the single layer model, the layers do not necessarily have any physical counterpart. The layers are conceptual objects with the sole purpose of absorbing and emitting energy, and the atmosphere of Venus is comprised of many of these layers. The thickness and distance between layers is ignored, as only intensive properties are relevant in this model. 


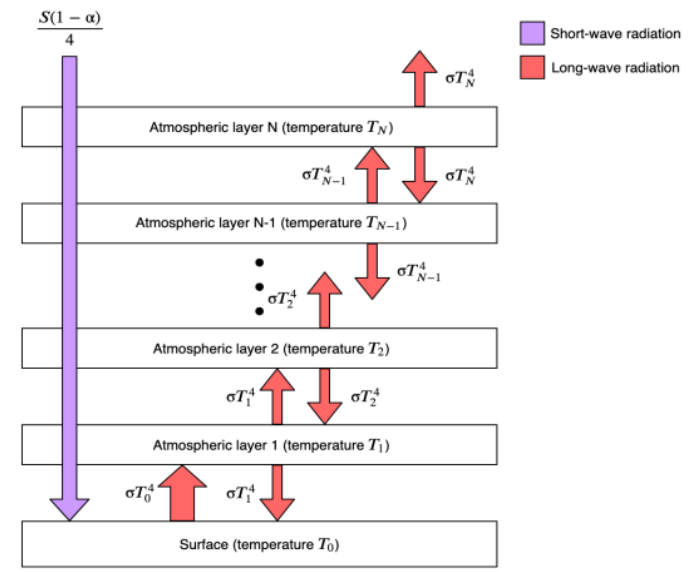

Fig. 2. A multiple atmospheric layer model of a planet at energy balance state. S represents the solar constant and $\alpha$ represents the surface albedo of the planet.

The equations from the one atmospheric layer model can be generalized to an N-layer model:

$$
\begin{gathered}
\sigma T_{N-1}^{4}=2 \sigma T_{N}^{4} \\
\sigma T_{N}^{4}+\sigma T_{N-2}^{4}=2 \sigma T_{N-1}^{4}
\end{gathered}
$$

We solve for an equation of the M-th layer:

$$
\sigma T_{M}^{4}=\frac{N-M+1}{N-M+2} \sigma T_{M-1}^{4}
$$

Assuming $\mathrm{M}=1$ :

$$
\sigma T_{1}^{4}=\frac{N}{N+1} \sigma T_{0}^{4}
$$

We will also use the energy balance equation of the surface of the planet:

$$
\sigma T_{0}^{4}=\sigma T_{1}^{4}+\frac{S(1-\alpha)}{4}
$$

Finally, solving for $\mathrm{T}_{0}$, we concluded that:

$$
T_{0}=\sqrt[4]{\frac{N+1}{4 \sigma} S(1-\alpha)}
$$

\section{Results and discussion}

\subsection{Planetary effective temperature model}

The classic planetary effective temperature model $T=$ $\sqrt[4]{\frac{S(1-\alpha)}{4 \sigma}}$ can serve as a baseline to compare how well the two model we developed work. (UCAR, center for science education) The predicted planetary effective temperatures, also known as the predicted blackbody temperatures, are displayed for Earth, Venus, and Mars (Table 1). While the model predicts Mars' temperature accurately, the actual temperatures of Earth and Venus are higher than the model's predictions. One of the assumptions of this model is that the planets do not have an atmosphere that produces a noticeable greenhouse effect, which is not the case and the source of the discrepancy for Earth and Venus.
Table 1. The facts and the predicted blackbody temperatures of planet Earth, Venus, and Mars. [6]

\begin{tabular}{|c|c|c|c|}
\hline & Earth & Venus & Mars \\
\hline $\begin{array}{c}\text { Solar flux } \\
(\mathrm{S})\end{array}$ & $\begin{array}{c}1361.0 \\
\mathrm{~W} / \mathrm{m}^{2}\end{array}$ & $\begin{array}{c}2601.3 \\
\mathrm{~W} / \mathrm{m}^{2}\end{array}$ & $\begin{array}{c}586.2 \\
\mathrm{~W} / \mathrm{m}^{2}\end{array}$ \\
\hline $\begin{array}{c}\text { Average } \\
\text { albedo }(\alpha)\end{array}$ & 0.306 & 0.77 & 0.250 \\
\hline $\begin{array}{c}\text { Actual } \\
\text { temperature } \\
(\mathrm{T})\end{array}$ & $288 \mathrm{~K}$ & $737 \mathrm{~K}$ & $210 \mathrm{~K}$ \\
\hline $\begin{array}{c}\text { Predicted } \\
\text { blackbody } \\
\text { temperature } \\
\text { (T') }\end{array}$ & $254.0 \mathrm{~K}$ & $226.6 \mathrm{~K}$ & $209.8 \mathrm{~K}$ \\
\hline $\begin{array}{c}\text { Discrepancy } \\
\text { (T-T') }\end{array}$ & $34 \mathrm{~K}$ & $510 \mathrm{~K}$ & $0 \mathrm{~K}$ \\
\hline
\end{tabular}

\subsection{One layer atmospheric layer model (Earth)}

The greenhouse gas atmosphere of Earth absorbs terrestrial radiation at certain wavelengths. Integration of the greenhouse gases absorption rate over the wavelengths yields an f value of 0.789 [7]. Then, we use the model $T=$ $\sqrt[4]{\frac{S(1-\alpha)}{4 \sigma\left(1-\frac{f}{2}\right)}}$ to calculate the surface temperature of Earth, considering the greenhouse effect. The modeled temperature is $\mathrm{T}=288 \mathrm{~K}$, the same as the actual temperature of the Earth, so the model is an improvement over the planetary effective temperature model and a success.

Figure 3 plots the entire range of $f$ values to show their respective predicted temperatures.

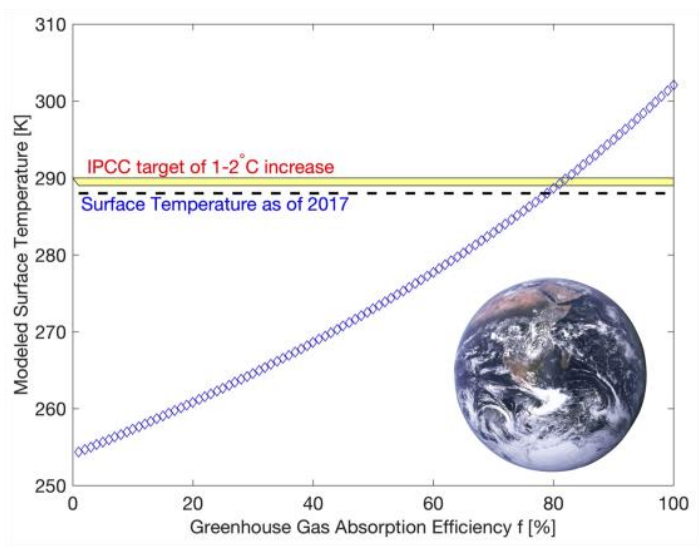

Fig. 3. The modeled surface temperature on Earth with greenhouse gas absorption efficiency range between $0-100 \%$. As of 2017, the surface temperature measured on Earth indicated the averaged absorption efficiency was $78.9 \%$. If greenhouse gas emissions remain uncontrolled, the surface temperature would soon exceed the IPCC target (yellow area). 


\subsection{Multiple atmospheric layer model (Venus)}

We can rearrange the equation $T=\sqrt[4]{\frac{N+1}{4 \sigma} S(1-\alpha)}$ to solve for the value of $\mathrm{N}$, which turns out to be $\mathrm{N}=111$. As shown in Figure 4, the surface temperature of an imaginary planet is increasing with atmospheric layer numbers. Using the actual temperature of Venus $\mathrm{T}=737$ $\mathrm{K}$, solar flux $2601.3 \mathrm{~W} / \mathrm{m}^{2}$, average albedo $\alpha=0.77$, and $\mathrm{N}=111$ in the model equation, we confirm that the predicted temperature is $T_{0}=737 \mathrm{~K}$. We can also conclude that Venus' atmosphere can be estimated using a 111 atmospheric layers model with each layer absorbing and emitting all of the long-wave radiation it receives.

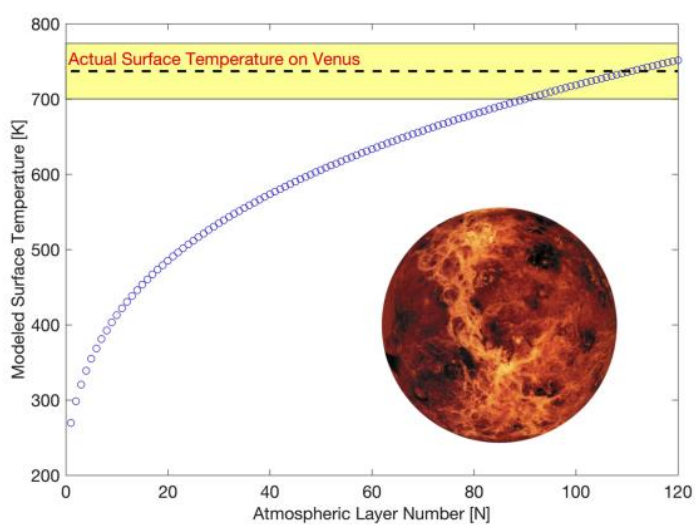

Fig. 4. Modeled surface temperature of a planet versus atmospheric layer number. The dashed line represents the actual surface temperature of Venus and the yellow area represents the $5 \%$ error range.

\subsection{Comparison of the greenhouse effect between Earth and Venus}

Earth and Venus have similar physical properties of mass and density reduced to the same pressure, different from each other by only $18.5 \%$ and $2 \%$ respectively [3]. However, Venus has a "thick" [3] atmosphere with 100\% cloud cover [4] and an albedo of 0.77 (NASA), much higher values compared to Earth's 50\% cloud cover [4] and albedo of 0.306 (NASA). Venus' cloud cover should have an effect of reducing Venus' global warming effect. On the other hand, due to Venus' proximity to the sun, it has a solar constant almost double that of the Earth, 2601.3 $\mathrm{W} / \mathrm{m}^{2}$ and $1361.0 \mathrm{~W} / \mathrm{m}^{2}$ respectively (NASA), which has an effect of increasing the blackbody temperature and effective temperature of Venus. The most likely reason for Venus having its much more severe greenhouse effect compared to Earth is the planets' difference in origin and evolution $[3,4]$.

There are noted differences in the distribution of several elements, including carbon dioxide $\left(\mathrm{CO}_{2}\right)$ and water $\left(\mathrm{H}_{2} \mathrm{O}\right)$. While Earth has most of its carbon stored in the mantle and carbonate rocks, Venus has most of its carbon dioxide in the atmosphere. While Earth's surface is abundant in water, Venus lacks surface water. Kaula concludes that the most likely theory regarding why Venus lacks surface water is that there have been no significant outgassing processes to release water within Venus [3].
To understand the difference between Venus and the Earth, we examined the early evolution of the temperature on each planet. Before Earth and Venus obtained their atmospheres, their surface temperatures were the same as their effective temperatures (albedo values of 0.15 are assumed for both planets because of the lack of clouds or ice). As water gradually outgassed from the interiors and accumulated in the atmosphere, the greenhouse effect of water vapor increased surface temperatures. On Earth, the saturation water vapor pressure of water was eventually reached as pressure buildup and at this point, the water started to precipitate to form the oceans, which could uptake the excessive amount of atmospheric $\mathrm{CO}_{2}$ and facilitate the fixation of carbon into carbonate rocks. In other words, over millions of years, Earth's carbon dioxide has been constantly removed by dissolving in rainwater, combining with minerals in rocks, running through rivers to the ocean, sedimenting in the sea-floor, and then being subducted to the mantle. On Venus, by contrast, the saturation water vapor pressure was never reached; oceans did not form and water vapor continued to accumulate in the atmosphere, resulting in a runaway greenhouse effect with a tremendous amount of $\mathrm{CO}_{2}$ stayed in the atmosphere [7]. Therefore, the Sun-Earth distance was critical in preventing this runaway greenhouse effect from happening on Earth.

\subsection{Global warming and its negative effects}

Global warming, causing temperature increases at an unprecedented rate, will inevitably result in numerous predictable and unpredictable changes within the biosphere. As shown in Figure 3, the modern atmosphere has an average absorption efficiency of $78.9 \%$, and if this value increases to $82.23 \%$, the surface temperature would reach $290 \mathrm{~K}$, which exceeds the IPCC target by the end of the 21 st century.

Global climate change has already had observable effects on the environment. Four of the major predicted changes, or changes that have already begun to take effect, include ocean acidification, sea-level rise, increased likelihood of droughts, and agricultural changes [2].

Oceans, taking up approximately 70 percent of the surface area of the Earth is one of the major carbon sinks and account for absorbing approximately 25 percent of carbon dioxide in the atmosphere [8]. Carbon dioxide molecules undergo a series of chemical reactions after being dissolved in the water [9]: 1). a molecule of carbon dioxide reacts with a molecule of water to form a molecule of carbonic acid $\mathrm{CO}_{2}+\mathrm{H}_{2} \mathrm{O} \leftrightarrow \mathrm{H}_{2} \mathrm{CO}_{3}$ (carbonic acid); 2). a hydrogen ion dissociates from the carbonic acid and forms a molecule of bicarbonate $\mathrm{H}_{2} \mathrm{CO}_{3} \leftrightarrow \mathrm{H}^{+}+\mathrm{HCO}_{3}^{-}$ (bicarbonate); 3 ). the disassociated hydrogen ion may react with carbonate ions in the ocean to form more bicarbonate $\mathrm{HCO}_{3}^{-} \leftrightarrow \mathrm{H}^{+}+\mathrm{CO}_{3}^{-2}$ (carbonate). The series of reactions show that carbon dioxide in ocean water is able to form $\mathrm{H}^{+}$ ions (reaction 2), which increases the acidity of the ocean, as well as uses up carbonate ions in the ocean (reaction 3). An increase in acidity is detrimental to numerous types of marine wildlife due to acid's corrosive property, while a decrease in the concentration of carbonate ions decreases 
the ability of calcifying organisms to form carbonate shells or skeletons. Coral reefs, ecosystems that contain reefbuilding coral, are one of the most biologically diverse ecosystems, yet are also one of the ecosystems that ocean acidification most severely affects [9, 10]. Corals build reefs made of calcium carbonate, and ocean acidification prevents new reefs from being formed and can even cause the deterioration of existing reefs [9].

The temperature increase of oceans, thermally conducted from the atmospheric temperature increase caused by global warming, has a two-fold effect: the volume of the oceans expand, due to the property of water expanding as temperature increases; higher temperatures also facilitate the melting of glaciers and polar ice, which effectively introduces new water molecules into the oceans [11]. The rising sea level can severely impact coastal cities and coastal terrestrial ecosystems through flooding. An accurate model of sea-level rise is hard to create due to the lack of complete understanding of the dynamics of melting glaciers and ice sheets. Vermeer and Rahmstorf proposed an improved model that correlates sea level rise with the mean global temperature increase, fitting empirical data nearly perfectly [12]. In addition, even if all greenhouse gas emissions were to be stopped, the global temperature increase and sea level rise will not immediately stop. Meehl et al. predict that the sea level will continue to rise, by a factor of 3 or more, 100 years after emissions have been stabilized [11].

Drought is one of the natural disasters that have an increased frequency due to climate change. Dai predicts "severe and widespread droughts" within the next century in various geographical areas, with the possibility of "persistent droughts" including the United States. The occurrences of drought is another difficult topic to model, as the influences of the El Niño-Southern Oscillation (ENSO) and changes in local and global sea surface temperature (SST) both affect the evaporation rate and precipitation rate of different areas [12, 13]. Dai has successfully developed a model that take ENSO and historical global aridity changes into account, and is able to accurately model global aridity changes up to 2010 [14].

Agriculture is a crucial component of modern society, and agricultural productivity is primarily dictated by aspects of climate such as temperature, precipitation, and natural disasters. Other agricultural factors that climate can modify include the presence of pests, availability of irrigation water, and occurrences of soil erosion. A potential positive effect of increased carbon dioxide concentrations is increased plant productivity, as photosynthesis uses carbon dioxide as a reactant [15]. Modeling agricultural changes in response to climate is complex since dynamic adaptations by farmers and consumers have to be taken into account, in order to avoid a "dumb farmer" model $[15,16]$. Adams et al. found that increasing temperatures diminishes crop yields while increasing precipitation increases crop yields [15].

\section{Conclusion}

This paper has examine two atmospheric models: the one atmospheric layer model and the multiple atmospheric layer model. Comparing the predicted surface temperatures of Earth and Venus to the actual surface temperatures recorded by NASA, it can be seen that the one atmospheric layer model is capable of accurately predicting the surface temperature of Earth and not Venus, while the multiple atmospheric layer model is capable of accurately predicting the surface temperature of Venus. The one atmospheric layer model predicts Earth to have an average absorption efficiency of $78.9 \%$, and the multiple atmospheric layer model predicts Venus to have 111 atmospheric layers with $100 \%$ absorption efficiencies. Given our understanding of the atmospheres of Earth and Venus, Venus demonstrates the effects of uncontrolled greenhouse gas emissions, and Earth's atmosphere seems to be approaching the condition of Venus' if greenhouse gas emission are also not controlled. The effects of climate change on Earth is already observable, especially in the areas of ocean acidification, sea-level rise, droughts, and agricultural. The effects of climate change on these areas and numerous others can be expected to worsen if greenhouse gasses increase the absorption efficiency of Earth's atmosphere, leading to an average higher surface temperature.

\section{References}

1. Hulme, M. (2016). $1.5{ }^{\circ} \mathrm{C}$ and climate research after the Paris Agreement. Nature Climate Change, 6, 222224.

2. Solomon, S., Qin, D., Manning, M., Chen, Z., Marquis, M., Averyt, K. B., ... \& Miller, H. L. (2007). The physical science basis. Contribution of working group I to the fourth assessment report of the intergovernmental panel on climate change, 2007, 235-337.

3. Kaula, W. M. (1990). Venus: A Contrast in Evolution to Earth. Science, 247(4947), 1191-1196.

4. Prinn, R. G., \& Fegley, B. (1987). The Atmospheres of Venus, Earth, and Mars: A Critical Comparison. Annual Review of Earth and Planetary Sciences, 15(1), 171-212.

5. Bohren, C. F., \& Clothiaux, E. E. (2006). Fundamentals of atmospheric radiation: an introduction with 400 problems. John Wiley \& Sons.

6. Williams, D. R. (2016, January 28). Planetary Fact Sheets. Retrieved from https://nssdc.gsfc.nasa.gov/planetary/planetfact.html

7. Jacob, D. J. (1999). Introduction to atmospheric chemistry. Princeton University Press.

8. Stocker, T. F., Qin, D., Plattner, G. K., Tignor, M., Allen, S. K., Boschung, J., ... \& Midgley, P. M. (2013). Climate change 2013: The physical science basis.

9. Hoegh-Guldberg, O., Mumby, P. J., Hooten, A. J., Steneck, R. S., Greenfield, P., Gomez, E., et al. (2007). Coral Reefs Under Rapid Climate Change 
and Ocean Acidification. Science, 318(5857), 17371742.

10. Pandolfi, J. M., Connolly, S. R., Marshall, D. J., \& Cohen, A. L. (2011). Projecting Coral Reef Futures Under Global Warming and Ocean Acidification. Science, 333(6041), 418-422.

11. Meehl, G. A., Washington, W. M., Collins, W. D., Arblaster, J. M., Hu, A., Buja, L. E., et al. (2005). How Much More Global Warming and Sea Level Rise? Science, 307(5716), 1769-1772.

12. Vermeer, M., \& Rahmstorf, S. (2009). Global sea level linked to global temperature. Proceedings of the National Academy of Sciences, 106(51), 2152721532.

13. Dai, A. (2011). Drought under global warming: a review. Wiley Interdisciplinary Reviews: Climate Change, 2(1), 45-65.

14. Dai, A. (2013). Increasing drought under global warming in observations and models. Nature Climate Change, 3(1), 52-58.

15. Adams, R. M., Hurd, B. H., Lenhart, S., \& Leary, N. (1998). Effects of global climate change on agriculture: an interpretative review. Climate Research, 11(1), 19-30.

16. Cline, W. R. (1996). The Impact of Global Warming of Agriculture: Comment. The American Economic Review, 86(5), 1309-1311. 